\title{
ILUSTRAÇÃO EMPÍRICA DA PERTINÊNCIA DA LONGITUDINALIDADE. CIDADANIA DOCENTE E DESEMPENHO DOS ESTUDANTES UNIVERSITÁRIOS
}

\author{
Arménio Rego \\ Universidade de Aveiro, Portugal
}

\section{INTRODUÇÃO}

\subsection{A pertinência da longitudinalidade}

Uma das grandes dificuldades colocadas pelos estudos correlacionais advem do facto de não ser possível delinear com algum rigor um nexo de causalidade entre as variáveis independentes e dependentes. Em Educação, o problema opera com especial acuidade em certos estudos que procuram explicar o desempenho académico dos estudantes. A questão coloca-se, muito simplesmente, do seguinte modo: são as variáveis independentes que explicam o desempenho, é este que explica as variáveis (pretensamente) independentes, ou os efeitos são recíprocos?

O paradigma desta possível ocorrência pode encontrar-se no caso particular da relação entre os comportamentos dos docentes e o (in)sucesso académico. Com efeito, é presumível que os comportamentos dos professores influenciem o desempenho dos seus estudantes. Mas também é plausível que estes desempenhos afectem as condutas docentes. A dificuldade agrava-se quando os comportamentos docentes são descritos pelos seus alunos. Nesse caso, pode dar-se a ocorrência de as descrições que os estudantes fazem dos comportamentos dos professores serem contaminadas pela classificação de desempenho académico que esses mesmos docentes Ihes atribuiram na disciplina. Assim, o investigador deve precaver-se da eventualidade de o estudante descrever o docente mais (des)favoravelmente quando a classificação obtida na disciplina é (des)favorável. Um dos modos de contornar o risco consiste em longitudinalizar o estudo, solicitando aos estudantes que descrevam o comportamento dos professores antes de terem conhecimento dos resultados da avaliação (e, o mais possível, alheados do contexto avaliativo).

O presente estudo envereda por um processo com este teor. O objectivo é o de mostrar como os comportamentos de cidadania docente (CIDOCE) dos professores universitários se relacionam com as notas dos respectivos alunos. Uma pesquisa anterior destinada a validar o instrumento de medida dos CIDOCE (Rego, 2001) havia revelado correlações significativas de razoável dimensão: 0.64 (comportamento empático), 0.47 (conscienciosidade pedagógica), 0.73 (cortesia) e 0.10 (uso correcto de acetatos). Exceptuando para o último (não significativo, para $p<0.5$ ), as significâncias foram sempre inferiores a 0.001 . Sucede que os estudantes inquiridos haviam descrito tais comportamentos de cidadania docente após conhecerem a nota na disciplina. A nossa hipótese é a de que estas correlações podem estar inflacionadas por via dessa opção 
metodológica. Afigura-se-nos necessário testar a relação com um procedimento que possa remover estes riscos. Por conseguinte, o presente estudo adopta uma metodologia longitudinal, faseada em dois momentos. A seguir, descreveremos o processo adoptado, e os resultados obtidos. Antes, todavia, explanaremos o contructo de cidadania docente, aplicável aos professores universitários.

\subsection{Cidadania organizacional e cidadania docente}

A cidadania docente pode ser definida a partir do conceito de "comportamentos de cidadania organizacional" (Smith, Organ \& Near, 1983; Organ, 1988, 1990, 1997; Van Dyne, Cummings \& Parks, 1995; Organ \& Ryan, 1995; Podsakoff, Ahearne \& MacKenzie, 1997; Netemeyer et al., 1997). A definição mais comummente utilizada é aquela que Organ (1988) propôs há mais de uma década: os comportamentos de cidadania organizacional são "os comportamentos discricionários, não directa ou explicitamente reconhecidos pelo sistema de recompensa formal, e que, no agregado, promovem o funcionamento eficaz da organização" (1988: 4).

Recentemente (Organ, 1997), ocorreu uma proposta de reconceptualização, de tal modo que a cidadania organizacional passou a ser definida como "desempenho contextual" (Borman e Motowidlo, 1997), reflectindo o conjunto de acções dos membros organizacionais que ajudam a modelar o contexto organizacional, social e psicológico em que as tarefas são levadas a cabo. São três os elementos caracterizadores destes actos: têm mais probabilidade do que outros de não fazerem parte do elenco de obrigações formais dos sujeitos; é também maior a probabilidade de não serem contratualmente (oficialmente) recompensados; contribuem para a eficácia das organizações.

Neste enquadramento conceptual, e numa tentativa de tradução do conceito para a vida docente, os comportamentos de cidadania docente dos professores universitários podem ser entendidos como as acções que ajudam a modelar o contexto organizacional, social e psicológico em que as tarefas formais dos agentes escolares se desenrolam (Borman e Motowidlo, 1997). A premissa que sustenta a grande maioria da extensa pesquisa dedicada à cidadania organizacional é a de que estes comportamentos contribuem para a eficácia das organizações. Existe, aliás, evidência empírica demonstrativa de que essa relação existe (e.g., Podsakoff \& MacKenzie, 1997). Por conseguinte, é igualmente legítimo admitir que os CIDOCE contribuem para a eficácia da instituição de ensino, designadamente o sucesso académico dos estudantes.

Os comportamentos de cidadania organizacional têm sido profusamente estudados (e.g., Organ, 1988, 1997; Organ \& Ryan, 1995; Van Dyne, Cummings \& Parks, 1995; Mackenzie, Podsakoff \& Ahearne, 1998), mas são escassas as investigações que se debruçam sobre os comportamentos de cidadania docente (Koh, Steers \& Terborg, 1995; Skarlicki \& Latham, 1995). O objectivo do presente estudo é precisamente o de mostrar como quatro dimensões de cidadania docente universitária se relacionam com o desempenho dos estudantes universitários. 


\subsection{Precauções metodológicas}

Deste objectivo deriva a necessidade de se enfatizarem os comportamentos presumivelmente mais significativos para a actividade dos estudantes: os que decorrem da relação entre professores e alunos, quer dentro quer fora da sala de aula. Embora a cidadania docente englobe actos de natureza científica (e.g., ajudar um colega a rever um artigo, emprestar documentação pertinente) e "administrativa" (e.g., substituir um colega na vigilância de um teste, assistir a reuniões não obrigatórias, apoiar voluntariamente os órgãos de gestão), importa pesquisar, sobretudo, os comportamentos de natureza pedagógica e relacional com os estudantes. Duas interrogações básicas se colocam.

Primeira: qual a fonte avaliadora dos CIDOCE mais adequada? Três hipóteses emergem: o próprio, os colegas, ou os alunos. Sucede que a auto-avaliação padece dos riscos inerentes ao ser-se "juiz em causa própria", afectando seriamente a objectividade. Por seu turno, a avaliação pelos colegas embate no facto de estes não poderem avaliar muitos dos actos executados pelos seus congéneres, especialmente os praticados em sala de aula, mas também os realizados em gabinete, ou mesmo nos "corredores" da instituição de ensino. Em contrapartida, a avaliação pelos alunos beneficia do facto de estes terem um conhecimento mais preciso dos comportamentos pedagógicos dos docentes, por via das experiências de relacionamento ao longo de um determinado período lectivo. Mas contém um risco não negligenciável: o de os juizos emitidos acerca do professor poderem ser influenciados pela nota obtida na disciplina.

Os dados disponíveis (Rego, 2001), recorrendo à avaliação pelos estudantes, denotam correlações significativas (e por vezes razoavelmente elevadas) entre quatro dimensões de CIDOCE (comportamento empático, conscienciosidade pedagógica, cortesia e uso de acetatos) e as classificações obtidas pelos estudantes. Todavia, a opção metodológica aí seguida comporta a possibilidade de estas relações estarem inflacionadas pelo facto de o aluno ter declarado a nota obtida na disciplina (variável dependente) ao mesmo tempo que se expressou acerca dos CIDOCE do docente respectivo (variáveis independentes). A questãochave não é propriamente a simultaneidade da obtenção dos dados, mas o facto de os alunos terem emitido juizos relacionados com as variáveis independentes quando já estavam na posse dos dados relativos à variável dependente. E esta é a segunda questão: como proceder para evitar esta possibilidade?

\section{METODOLOGIA}

Foram estes riscos e dificuldades metodológicas que a longitudinalidade procurou minimizar. Solicitámos, então, a uma amostra de 249 estudantes da Universidade de Aveiro (oriundos de 27 curos; $37 \%$ do sexo feminino) que cotassem os CIDOCE antes do processo de avaliação se iniciar. Adoptou-se o seguinte procedimento, em duas fases. Na primeira, obtivemos os dados relativos aos comportamentos de cidadania 
dos docentes. Na segunda, recolhêmos a informação respeitante às classificações dos estudantes. As datas de recolha dos dados foram escolhidas de acordo com dois critérios:

a) O período de contacto entre aluno e professor deveria ser suficientemente grande para que o estudante pudesse dispor de um período de convivência pedagógica significativo, e ter um grau de conhecimento razoável dos comportamentos do professor.

b) O aluno deveria estar suficientemente distanciado do período de avaliação, de tal modo que os seus julgamentos acerca dos CIDOCE não fossem contaminados pelo contexto avaliativo.

Por conseguinte, optou-se por medir os CIDOCE durante a penúltima semana de aulas (1 $1^{\text {a }}$ semana de Janeiro). Os testes de avaliação decorreram no período que medeia entre 18/1 e 24/2. Após a data limite de lançamento das classificações, os alunos foram abordados no sentido de nos informarem da classificação obtida. Essa constituiu a segunda fase.

Na fase 1 , todos os estudantes foram abordados directa e pessoalmente. Foi-lhes solicitada colaboração para uma pesquisa, tendo-lhes sido explicado que pretendíamos que avaliassem, anonimamente, um professor à sua escolha. Uma restricção foi colocada: a disciplina em causa deveria ser leccionada apenas por esse docente. Com efeito, não seria metodologicamente correcto relacionar a) o desempenho académico "gerado" pela concomitância da leccionação de vários professores com b) os CIDOCE de um só docente.

De seguida, foi-lhes solicitado que respondessem ao questionário de medida dos CIDOCE. Trata-se de um instrumento anteriormente testado (Rego, 2001), composto por 32 escalas tipo Likert, em que o estudante é convidado a assinalar, numa escala de 7 pontos (1: "nunca"; ...; 7: "sempre"), a frequência com que o professor adopta os comportamentos inerentes aos itens. A factorialização dos dados (Catell, 1966; Ford et al., 1986; Kline, 1994; Kim \& Mueller, 1978a, 1978b) havia sugerido a existência de qutro dimensões de cidadania (Rego, 2001): comportamento empático (16 itens; alpha: 0.97), conscienciosidade pedagógica (8 itens; 0.92), cortesia (6 itens, 0.90 ) e uso adequado de acetatos (2 itens, 0.81 ).

A fase 2 iniciou-se dois dias após a data limite estipulada para o lançamento das classificações, o que corresponde a cerca de dois meses após o termo da primeira fase. Enviaram-se a todos os respondentes os sobrescritos que haviam sido preparados na primeira fase. Foi-lhes solicitado que assinalassem a classificação obtida na disciplina leccionada pelo professor avaliado. Um sobrescrito de RSF foi-lhes proporcionado. Dos 249 respondentes da fase 1, devolveram a resposta 142 estudantes (índice de respostas: 56\%). Provinham de 24 cursos, e a sua distribuição por sexos era de $43 \%$ para o sexo feminino e $57 \%$ para o masculino. Atendendo ao anonimato, a conexão entre as respostas das duas fases foi possível graças a um código alfanumérico inventado pelos estudantes. 


\section{RESULTADOS}

Em primeiro lugar, os dados referentes aos CIDOCE foram sujeitos a uma análise factorial confirmatória (Byrne, 1998; Joreskog \& Sorbom, 1993). Os dois itens referentes ao último factor (uso de acetatos) emergente da análise exploratória foram excluidos (Byrne, 1998; Joreskog \& Sorbom, 1993). Os índices de ajustamento do modelo tri-partido (integrando os 30 itens que mais saturavam os três primeiros factores obtidos na análise exploratória) revelaram valores insatisfatórios. Numa tentativa de obter uma estrutura factorial mais ajustada aos dados, retirámos alguns itens (Byrne, 1998), tendo os índices melhorado substancialmente. Finalmente, testámos um modelo em que o primeiro factor foi desdobrado em dois, de acordo com o conteúdo semântico dos itens.

Quadro I - Índices de ajustamento de vários modelos factoriais (escalas CIDOCE)

\begin{tabular}{lccc}
\hline & $\begin{array}{c}\text { Modelo de 3 factores } \\
\text { com os 32 itens } \\
\text { originais } \\
\text { (Rego, 2001) } \\
(\mathbf{n = 2 4 9 )}\end{array}$ & $\begin{array}{c}\text { Modelo de 3 factores } \\
\text { com 14 itens }\end{array}$ & $\begin{array}{c}\text { Modelo de 4 factores } \\
\text { com 14 itens }\end{array}$ \\
\hline Qui-quadrado (p) & $966.08(0.0000)$ & $134(0.00002)$ & (n=249) \\
Qui-quadrado/graus de & 3.2 & 1.8 & $91.65(\mathrm{p}=0.05)$ \\
liberdade & 0.096 & 0.057 & 1.29 \\
RMSEA & 0.77 & 0.93 & 0.034 \\
GFI & 0.73 & 0.90 & 0.95 \\
AGFI & 0.65 & 0.65 & 0.93 \\
PGFI & 0.79 & 0.94 & 0.64 \\
NFI & 0.83 & 0.97 & 0.96 \\
NNFI & 0.72 & 0.76 & 0.99 \\
PNFI & 0.85 & 0.97 & 0.75 \\
CFI & 0.85 & 0.97 & 0.99 \\
IFI & 0.77 & 0.92 & 0.99 \\
RFI & 95.85 & 208.51 & 0.94 \\
N crítico & & & 286.88 \\
\hline
\end{tabular}

Este modelo factorial tetra-partido, composto por 14 itens, denotou os melhores índices de ajustamento (quadro I). Os factores representam quatro categorias de comportamento que designámos de "comportamento participativo", "orientação prática", "conscienciosidade pedagógica" e "(des)cortesia". Os itens que as compõem, e a estrutura factorial confirmatória estão expostos no quadro II. Aí se assinalam, também, os resultados da análise operada sobre os dados de uma amostra inquirida numa das fases da pesquisa exploratória (Rego, 2001).

O "comportamento participativo", representa o fomento da participação dos estudantes no processo pedagógico. A "orientação prática", reflecte a orientação pedagógica do docente para ilustrar a explanação das matérias com exemplos práticos. A "conscienciosidade pedagógica" consubstancia comportamentos reveladores de uma postura preocupada com o exercício rigoroso da função docente (e.g., preparar bem as 
aulas, ser exigente, dar as aulas com rigor e seriedade. A "(des)cortesia" reflecte um tratamento (des)respeitador dos estudantes.

Quadro II - Análise factorial confirmatória do instrumento de medida dos CIDOCE (Lambdas correspondentes à solução completamente standardizada)

\begin{tabular}{|c|c|c|}
\hline & $\begin{array}{l}\text { Dados do } \\
\text { presente estudo } \\
(n=249)\end{array}$ & $\begin{array}{c}\text { Dados de estudo } \\
\text { exploratório } \\
\text { anterior } \\
\text { (Rego, 2001) } \\
(n=146)^{\star *}\end{array}$ \\
\hline Comportamento participativo* & $(0.85)$ & $(0.90)$ \\
\hline Fomenta a participação dos alunos na aula. & 0.81 & 0.80 \\
\hline Dialoga abertamente com os alunos. & 0.79 & 0.89 \\
\hline Quando pede a opinião dos alunos ... toma as ideias deles em consideração. & 0.81 & 0.90 \\
\hline Orientação prática* & $(0.85)$ & $(0.87)$ \\
\hline Os exemplos que dá na aula são interessantes para a vida dos alunos. & 0.80 & 0.82 \\
\hline Nas aulas, alerta para o sentido prático das coisas. & 0.84 & 0.90 \\
\hline Ilustra a exposição da matéria com exemplos práticos. & 0.77 & 0.95 \\
\hline Conscienciosidade pedagógica & $(0.88)$ & $(0.94)$ \\
\hline Expõe a matéria de modo organizado. & 0.90 & 0.92 \\
\hline Nas aulas, não segue um fio de pensamento (i.e., "mistura tudo"). (I) & -0.78 & -0.85 \\
\hline Prepara bem as aulas. & 0.86 & 0.91 \\
\hline É metódico na exposição da matéria & 0.71 & 0.89 \\
\hline (Des)cortesia & $(0.82)$ & $(0.88)$ \\
\hline Culpa os alunos pelos maus resultados.(I) & 0.67 & 0.78 \\
\hline Marginaliza os alunos de que não gosta.(I) & 0.80 & 0.88 \\
\hline Trata com indiferença os alunos menos bons.(I) & 0.74 & 0.74 \\
\hline $\begin{array}{l}\text { Quano os alunos vão ao gabinete para esclarecer dúvidas ... faz troça dos } \\
\text { erros...(I) }\end{array}$ & 0.75 & 0.89 \\
\hline \multicolumn{3}{|c|}{$\begin{array}{l}\text { Para o cálculo das cotações finais nas dimensões factoriais emergentes, as pontuações nestes itens foram invertidas: o } 7 \text { passou } \\
\text { a } 1 \text {, e vice-versa; o } 6 \text { passou a } 2 \text {, e vice-versa; e por aí adiante. } \\
\text { ntesis: coeficientes alpha de Cronbach. } \\
\text { lois factores resultam do desdobramento do primeiro factor (comportamento empático) obtido na análise factorial } \\
\text { ria (Rego, 2001). } \\
\text { de ajustamento: GFI: } 0.90 ; \text { AGFI: } 0.85 ; \text { CFI: } 0.98 ; \text { IFI: } 0.98\end{array}$} \\
\hline
\end{tabular}

(I)

* Estes dois factores resultam do desdobramento do primeiro factor (comportamento empático) obtido na análise factorial exploratória (Rego, 2001).

** Índices de ajustamento: GFI: 0.90; AGFI: 0.85; CFI: 0.98; IFI: 0.98

O quadro III expõe as médias, desvios-padrão e correlações entre variáveis. As relações entre as variáveis de cidadania são moderadas e significativas. Mais modestas e nem sempre significativas são as relações entre os CIDOCE e as classificações, pois apenas o coeficiente relativo à cortesia é estatisticamente significativo. Não 
se detectaram diferenças significativas, nestas relações, entre os estudantes de cada sexo. Para efeitos comparativos, o quadro apresenta igualmente os coeficientes de correlação (entre os CIDOCE e as notas) obtidos com uma amostra inquirida no âmbito do estudo exploratório (Rego, 2001). Nesse estudo, os estudantes descreveram o comportamento dos professores já na posse da classificação obtida na disciplina. Os valores são significativamente mais elevados do que os emergentes do actual estudo.

Quadro III - Médias, desvios-padrão e correlações entre variáveis

\begin{tabular}{|c|c|c|c|c|c|c|c|}
\hline & Média & DP & 1 & 2 & 3 & 4 & 5 \\
\hline 1. Comportamento participativo & 4.1 & 1.5 & & & & & \\
\hline 2. Orientação prática & 4.3 & 1.6 & $0.74^{\star \star \star}$ & & & & \\
\hline $\begin{array}{l}\text { 3. Conscienciosidade } \\
\text { pedagógica }\end{array}$ & 4.8 & 1.2 & $0.34^{\star * *}$ & $0.39^{* * *}$ & & & \\
\hline 4. Cortesia & 5.1 & 1.5 & $0.44^{* * *}$ & $0.35^{\star * *}$ & $0.38^{* * *}$ & & \\
\hline 5. CIDOCE global(a) & 5.6 & 1.4 & $0.84^{\star \star \star}$ & $0.82^{\star \star \star}$ & $0.69^{\star \star *}$ & $0.70^{\star \star \star}$ & \\
\hline 6. Classificação na disciplina(b) & 12.4 & 3.1 & $\begin{array}{l}0.18^{*} \\
\left(0.59^{* * *}\right)\end{array}$ & $\begin{array}{l}0.18^{*} \\
\left(0.65^{\star * *}\right)\end{array}$ & $\begin{array}{l}-0.05 \\
\left(0.49^{\star * *}\right)\end{array}$ & $\begin{array}{l}0.35^{\star * *} \\
\left(0.60^{\star * *}\right)\end{array}$ & $\begin{array}{l}0.21^{*} \\
\left(0.64^{* * *}\right)\end{array}$ \\
\hline
\end{tabular}

${ }^{*} \mathrm{p}<0.05{ }^{* *} \mathrm{p}<0.01 \quad{ }^{* * *} \mathrm{p}<0.001$
(a) Média das cotações nas quatro categorias de cidadania (alpha $=0.77$ )

(b) Foram retirados da análise os 21 estudantes que declararam não comparecer a exame, pelo que o ${ }^{\circ}$ de indivíduos, nesta variável, é 121.

Entre parêntesis: correlações obtidas com amostra $(n=60)$ inquirida no âmbito do estudo exploratório $($ Rego,2001).

O quadro IV representa os resultados das regressões hierárquicas. Mostra qual a variância (na classificação obtida pelos estudantes) explicada por cada variável de cidadania depois de estimada a variância explicada pelas restantes três variáveis. Verifica-se que apenas a cortesia denota potencial explicativo relevante e significativo. De qualquer modo, a variância total explicada atinge o modesto valor de $15 \%$.

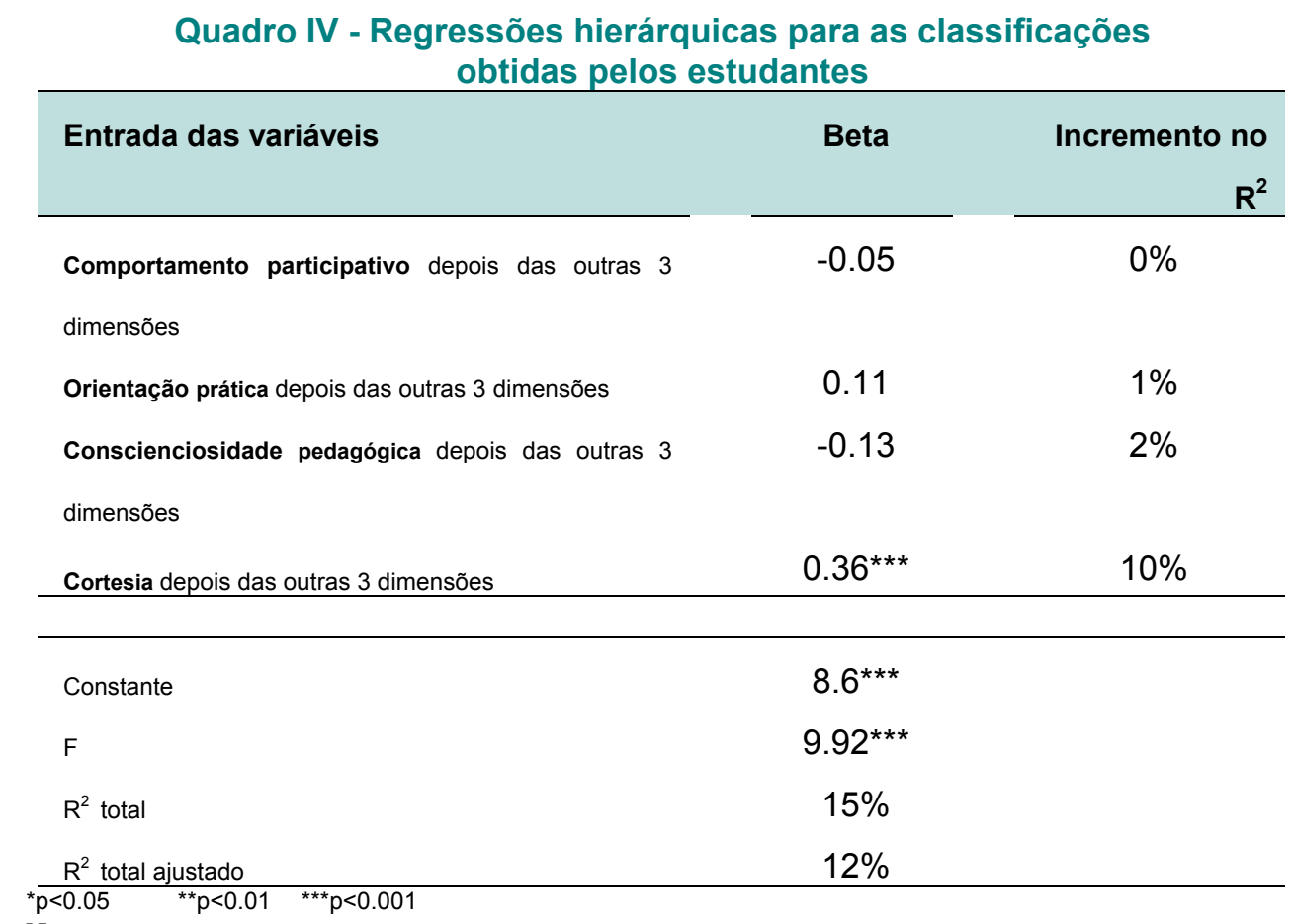

NOTA : os Betas, a constante e o valor do F referem-se à equação de regressão contendo as quatro dimensões de CIDOCE 


\section{DISCUSSÃO E CONCLUSÕES}

Os resultados sugerem que existe uma dimensão de CIDOCE com potencial explicativo para a nota obtida pelo estudante: a cortesia. A variância explicada, embora modesta, não é despicienda, e induz a necessidade de se aprofundar o estudo do impacto que os comportamentos dos docentes têm sobre os desempenhos académicos dos seus alunos. É presumível que os professores (des)corteses exerçam um efeito (des)motivador sobre os seus estudantes, induzindo-os a alterar os seus níveis de esforço e empenhamento, com repercussões no desempenho académico.

Sucede que as relações aqui obtidas foram bastante inferiores às emergentes no estudo exploratório anteriormente efectuado (Rego, 2001). Este dado é suficiente para conferir verosimilhança à hipótese de os estudantes procederem às descrições comportamentais dos seus docentes sob a influência da classificação que esses mesmos docentes Ihes atribuiram. E, numa perspectiva mais geral, sugerem a extrema importância de se adoptarem metodologias que acautelem essa ocorrência, designadamente longitudinais.

A longitudinalidade não resolve, porém, todas as dificuldades metodológicas. Embora reduza fortemente os riscos de os estudantes poderem notar os seus docentes debaixo da influência da nota que estes lhes atribuiram na disciplina, não nos garante a inexistência de relações espúrias, nem nos permite projectar nexos de causalidade óbvios:

a) No caso da espuriedade, isto significa que a relação detectada pode resultar da influência de uma terceira variável sobre os dois tipos de variáveis aqui pesquisadas, e não propriamente do efeito dos comportamentos dos docentes sobre o trabalho e desempenho dos estudantes. Na verdade, a relação entre cortesia e classificações pode resultar do facto de alguns professores denotarem características de personalidade que os induzem a ser mais corteses e, simultaneamente, mais benevolentes na atribuição das classificações.

b) No que concerne às relações de causa-efeito, é bastante plausível que sejam os CIDOCE a influenciar as classificações dos estudantes. Mas a hipótese inversa também contém alguma verosimilhança: pode suceder que os docentes reajam com mais cortesia aos estudantes que, pelas suas qualidades, empenho, dedicação e relacionamento pedagógico, sejam potencialmente mais eficazes do ponto de vista do desempenho académico. É ainda possível que se conjuguem efeitos recíprocos. 
É necessário, por conseguinte, prosseguir as pesquisas, quer através de estudos que testem a existência da eventual espuriedade, quer por via de metodologias experimentais que permitam testar com mais rigor as eventuais relações de causa-efeito. Há, por outro lado, todo o interesse científico em replicar o estudo noutras instituições de ensino superior, e comparar os resultados para diferentes tipos de cursos (e.g., tecnológicos versus humanísticos). Réplica idêntica pode ser concretizada noutros níveis de ensino, embora depois de introduzidas alterações apropriadas no instrumento de medida dos CIDOCE (Rego \& Sousa, 1998, 1999).

\section{BIBLIOGRAFIA}

BORMAN, W. C.; MOTOWIDLO, S. J. (1997). Task performance and contextual performance - the meaning for personnel selection research. Human Performance, 10: 99-109.

BYRNE, B. M. (1998). Structural Equation Modeloing with Lisrel, Prelis, and Simplis. London: Lawrence Erlbaum Associates

CATTELL, R. B. (1966). The meaning and strategic use of factor analysis. In R. B. Cattell (Ed.), Handbook of Multivariate Experimental Psychology. Chicago: Rand McNally.

FORD, J. K.; MACCALLUM, R. C.; TAIT, M. (1986). The application of exploratory factor analysis in applied psychology: A critical review and analysis. Personnel Psychology, 39: 291-314.

JORESKOG, K.; SORBOM, D. (1993). Lisrel 8: Structural Equation Modeling with the Simplis Command Language. Scientific Software International.

KIM, J.; MUELLER, C. W. (1978a). Factor analysis: Statistical methods and practical issues. Beverly Hills, CA: SAGE.

KIM, J.; MUELLER, C. W. (1978b). Introduction to factor analysis - what it is and how to do it. Beverly Hills, CA: SAGE.

KLINE, P. (1994). An easy guide to factor analysis. London: Routledege.

$\mathrm{KOH}, \mathrm{W}$;; STEERS, R.; TERBORG, J. (1995). The effects of transformational leadership on teacher attitudes and student performance in Singapore, Journal of Organizational Behavior, 16, 319-333.

MACKENZIE, S. B.; PODSAKOFF, P. M.; AHEARNE, M. (1998). Some Possible Antecedents and Consequences of In-Role and Extra-Role Salesperson Performance. Journal of Marketing, 62(July): 87-98.

NETEMEYER, R. G.; BOLES, J. S.; MCKEE, D.; MCMURRIAN, R. (1997). An Investigation Into the Antecedents of Organizational Citizenship Behaviors in a Personal Selling Context. Journal of Marketing, 61: 8598.

ORGAN, D. W.; RYAN, K. (1995). A Meta-Analytic Review of Attitudinal and Dispositional Predictors of Organizational Citizenship Behavior. Personnel Psychology, 48: 775-802.

ORGAN, D. W. (1988). Organizational Citizenship Behavior: the Good Soldier Syndrome. Lexington, MA: Lexington Books.

ORGAN, D. W. (1990). The Motivational Basis of Organizational Citizenship Behavior. In B. M. Staw \& L. L. Cummings (Eds.). Research in Organizational Behavior (vol. 12, pp. 43-72). Greenwich, Connecticut: JAI Press.

ORGAN, D. W. (1997). Organizational Citizenship Behavior - its construct clean-up time. Human Performance, 10: 85-97. 
PODSAKOFF, P. M.; MACKENZIE, S. B. (1997). Impact of Organizational Citizenship Behavior on Organizational Performance - a Review and Suggestions for Future Research. Human Performance, 10(2): 133151.

PODSAKOFF, P. M.; AHEARNE, M.; MACKENZIE, S. B. (1997). Organizational Citizenship Behavior and the Quantity and Quality of Work Group Performance. Journal of Applied Psychology, 82: 262-270.

REGO, A.; SOUSA, L. (1998). Comportamentos de cidadania do professor. Rumos, 22: 18-19.

REGO, A.; SOUSA, L. (1999). Comportamentos de cidadania do professor: sua importância na comunidade escolar. Revista de Educação, VIII(1): 57-63.

REGO, A. (2001). Comportamentos de cidadania docente universitária: operacionalização de um construto. Revista de Educação, X(1): 87-98.

SKARLICKI, D. P.; LATHAM, G. P. (1995). Organizational Citizenship Behavior and Performance in a University Setting. Canadian Journal of Administrative Sciences, 12(3): 175-181.

SMITH, C. A.; ORGAN, D. W.; NEAR, J. P. (1983). Organizational Citizenship Behavior: Its Nature and Antecedents. Journal of Applied Psychology, 68: 653-663.

VAN DYNE, L.; CUMMINGS, L. L.; PARKS, J. M. (1995). Extra-role Behaviors: In Pursuit of Construct and Definitional Clarity (a Bridge over Muddied Waters). In L. L. Cummings \& B. M. Staw (Eds.), Research in Organizational Behavior: (vol. 17, pp. 215-285). Greenwich, Connecticut: JAI Press. 


\title{
Contactar
}

Revista lberoamericana de Educación

\author{
Principal OEI
}

